Iraqi Journal of Information and Communications Technology(IJICT)

Conference Series: The $1^{\text {st }}$ Conference of Applied Researches in Information Engineering(ARIE2021), 2021

ISSN:2222-758X e-ISSN:2789-7362

\title{
PERFORMANCE EVALUATION OF FILTER BANK MULTI CARRIER MODULATION IN MULTIPATH FADING CHANNELS
}

\author{
Ali A. Abdulhussein ${ }^{1}$, Hikmat N. Abdullah ${ }^{2}$ \\ 1,2 College of Information Engineering, Al-Nahrain University, Baghdad, Iraq \\ Ali.adnan.itc91@gmail.com ${ }^{1}$, hikmat.abdullah@ coie-nahrain.edu.iq ${ }^{2}$ \\ Received:7/5/2021, Accepted:22/6/2021
}

\begin{abstract}
Filter Bank Multi-Carrier (FBMC) modulation is one of the most significant enablers for future 5G technologies. It is a modulation technique for resolving inter-carrier and inter-symbol interference using two possible methods: Frequency Spreading (FS) and Poly Phase (PP) implementation. Cyclic prefixes are used in OFDM for signal robustness, but they have some disadvantages in orthogonal frequency division multiplexing. FBMC is used to solve the disadvantages of OFDM and save bandwidth. In this paper, performance comparisons in terms of Bit error rate between OFDM and FBMC systems in AWGN as well as 5G channel which is SUI-6 multipath fading channel at the different number of subcarriers are presented. The obtained results show that FBMC over-performs OFDM in the SUI-6 channel and the improvement margin reaches $20 \%$. Also, the results show that the improvement margin is increased as the number of subcarriers decreased.
\end{abstract}

keywords: Filter bank multi-carrier, 5G, SUI-6 channel, Spectral efficiency, Subcarrier.

\section{INTRODUCTION}

The most widely used wireless broadband networking technology is Orthogonal Frequency Division Multiplexing (OFDM). Its architecture simplicity is aided by the use of the Inverse Fast Fourier Transform (IFFT) as a modulator and the Fast Fourier Transform (FFT) as a demodulator. Despite its many benefits, OFDM has several drawbacks, including spectral inefficiency caused by the cyclic prefix, which is considered a waste of bandwidth. Currently, research areas are looking into other options to address the aforementioned deficiency to meet the ever-increasing demand for throughput. To achieve this goal and without losing the privilege of simple practical design, researchers have turned their attention to other technologies such as the Filter Bank Multicarrier (FBMC) modulation scheme, which can meet the previously stated spectral requirements with some additional signal processing [1]. FBMC is being heavily considered as the physical layer solution for $5 \mathrm{G}$ communication systems which has to attract features including end-to-end round-trip latency of 1 millisecond, 1000 $\times$ bandwidth is available, perceived availability of 99.999 percent, the impression of complete coverage, network energy consumption is decreased by 9\%, low-power, and resistance to the channel effects [2]. Chang proposed the primarily FBMC system, which was further developed by Saltzberg in about 1965, and it was rediscovered by Bellanger to be proposed as an option for potential wireless communications [3]. FBMC has real data symbols whereas most communication systems follow the QAM as the input modulation scheme to the multicarrier transmission system. This has led to the new definition of Offset Quadrature Amplitude Modulation (OQAM) [4]. The basic concept behind FBMC is that the time-domain symbol length is increased and a prototype filter is used to filter it. To compensate for the data rate loss caused by the expanded symbols, the symbols overlap in both the frequency and time domains, allowing the original data rate to be retained. An easy alternative is to use an expanded IFFT with Frequency Spreading (FS), which uses frequency-domain symbol construction.

https://ijict.edu.iq 
Iraqi Journal of Information and Communications Technology(IJICT)

Conference Series: The $1^{\text {st }}$ Conference of Applied Researches in Information Engineering(ARIE2021), 2021

ISSN:2222-758X e-ISSN:2789-7362

\section{Related Works}

In [1] , various FBMC implementations are compared in terms of complexity and quantization error. In [4] , different aspects of FBMC like the complexity of implementation, channel estimation-equalization, synchronization, and application in Cognitive Radio (CR) are investigated. In [5] FBMC-OFDM is explained in detail using different types of polyphase filters including synthesis polyphase filters and analysis polyphase filters. In [6], the performance difference between FBMC-OQAM and OFDM is studied by building the system model, and the effect of the overlap factor of the prototype filter on the performance of multi-carrier technology in the FBMC-OQAM system is also studied. In [7] , a performance comparison between two different multi-carrier transmission techniques: OFDM, based on the FFT and cyclic prefix (CP) addition; and FBMC (Filter Bank Multicarrier), which, as indicated by its name, is based on a filter bank architecture is presented. In [8], some of the critical challenges in FBMC systems to make them viable for practical applications are addressed. In [9] , FBMC modulation techniques are compared with the existing OFDM technique in terms of Spectral Density, Spectral Efficiency, PAPR, BER of FBMC, and OFDM modulation techniques to analyze their merits. In [10], an overview of FBMC, with emphasis on the features which impact communication networks are provided. This work is an attempt to provide fair comparisons of the Bit error rate of OFDM and FBMC in the presence of an important type of transmission channel.

\section{SYSTEM MODEL}

A channel bank multicarrier is a way to solve some of the shortcomings of OFDM, or orthogonal repeat division multiplexing. One of the major flaws stems from the fact that OFDM necessitates the use of a so-called cyclic prefix. Fig. 1 shows the block diagram of the FBMC system.

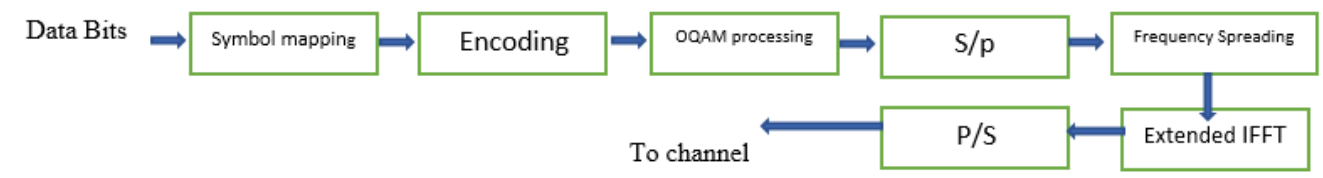

(a)

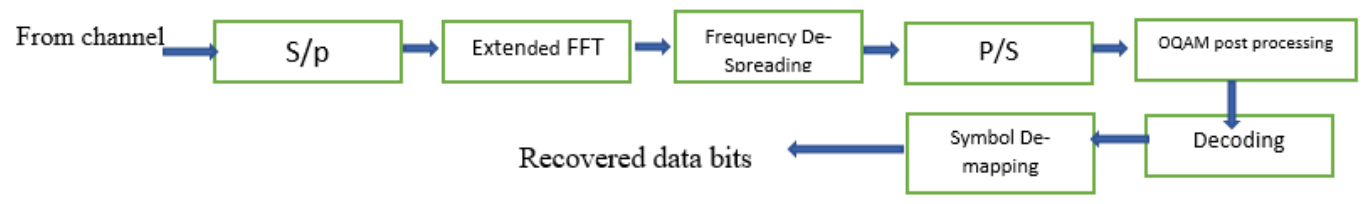

(b)

Figure 1: FBMC communication system (a) Transmitter, (b) Receiver

At the transmitter side, first, the input data bits enter the symbol mapping where the modulation symbol map generates M-QAM modulated electrical signals, and the modulation symbol de-mapper demodulates the signals based on the form of modulation used. The modulation type of the symbol mapper matches the modulation type of the mapper [5], OQAM is used to modulate symbols after channel coding and symbol mapping of serial high-speed files. The aim of OQAM 
preprocessing is to maintain subcarrier orthogonality. To become transmission symbols, OQAM pretreatment processes complex symbols in both the actual and virtual parts and interlaces half a symbol cycle in the time interval. Subcarriers are created by dividing the real and imaginary parts of the interleaved delay in this way. Any subcarriers and neighboring subcarriers have an orthogonal distribution at sampling time. The transmission symbols are then subjected to an IFFT operation, after which the prototype filter banks with various offsets are screened. Finally, the time-domain synthesized signals are superimposed and sent out [6]. At the receiver side, a collection of prototype filters is also used, which are symmetrical and have the same output as the prototype filter banks at the transmitter. The original signal is first filtered using prototype filter banks with various offsets. FFT and OQAM are then used to restore the original signal. OQAM post-processing involves taking the real part of the signal modulated to the subcarrier and reconstructing the real signal into a complex signal using the mutual conversion of the real and complex numbers [7] .

\section{OQAM Processing And Post-Processing}

The key idea behind the FBMC/OQAM scheme is to send real (offset QAM) symbols rather than complex (QAM) symbols as in OFDM. If each FBMC block contains M FBMC symbols and each symbol has $\mathrm{N}$ subcarriers in the frequency domain, one FBMC block will transmit MN modulated QAM symbols in total [8] . Fig. 2 depicts the structure of FBMC/OQAM. The signal is modulated by QAM to become a complex signal in the FBMC-OQAM scheme, and then the real and imaginary parts of the complex signal are taken separately. The imaginary part of the signal is half a cycle later than the real part, and it is then converted into a parallel signal using series-parallel conversion, resulting in the real and imaginary parts of each symbol being transmitted on subcarriers. After that, the signal is modulated by a prototype and phase filter, and each subcarrier signal is superimposed on the final transmitted signal. Different channels are isolated at the receiving end by spectrum shifting. The real and imaginary parts of each signal are divided. The original process is restored after a matched filter [9].

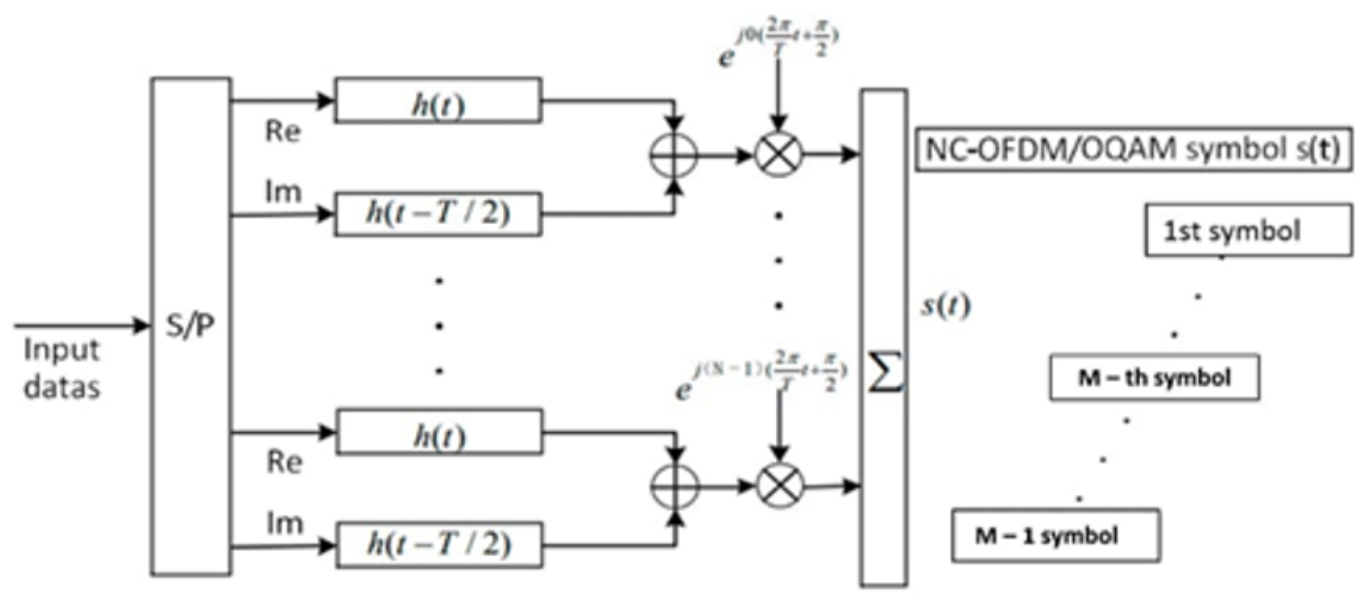

Figure 2: Block diagram of FBMC/OQAM system 
Suppose that the FBMC-OQAM system has $\mathrm{N}$ subcarriers, which are modulated by OQAM and serial-to-parallel conversion, it is converted into matrix $\mathrm{X}$ which is defined as $X=\left(X^{0}, X^{1}, X^{m}, \ldots, X^{M-1}\right)^{T}$, where $\mathrm{m}$ denotes the $\mathrm{m}$-th data block and $\mathrm{M}$ denotes the symbol size. $X=\left(x_{0}^{m}, x_{1}^{m}, x_{k}^{m}, \ldots, x_{N-1}^{m}\right)$, where $\mathrm{k}$ represents subcarrier index, $x_{k}^{m}$ represents data on the $\mathrm{k}$ subcarrier and the $\mathrm{m}$ symbol block $x_{k}^{m}=a_{k}^{m}+j b_{k}^{m}$ where $a_{k}^{m}, b_{k}^{m}$ represent the real and imaginary parts of the signal. $b_{k}^{m}$ lags $a_{k}^{m} T / 2$ in the time domain, where T represents the QAM symbol period. The signal through the prototype filter $\mathrm{h}(\mathrm{t})$ is represented as:

$$
x_{k}^{m}(t)=\left[a_{k}^{m} h(t-m T)+j b_{k}^{m} h(t-T / 2-m T)\right] e^{j \frac{\pi}{2} k}, k=0,1, \ldots, N-1
$$

After N subcarriers orthogonal modulation:

$$
S_{k}^{m}(t)=\left[a_{k}^{m} h(t-m T)+j b_{k}^{m} h(t-T / 2-m T)\right] e^{j\left(2 \frac{\pi}{T} t+\frac{\pi}{2}\right) k}, k=0,1, \ldots, N-1
$$

The signal on the m-th data block can be represented as:

$$
S^{m}(t)=\sum_{k=0}^{N-1} S_{k}^{m}(t) ; m T \leq t \leq m T+L+\frac{T}{2}
$$

$\mathrm{L}$ denotes the length of the filter, where L=KM. Finally, $\mathrm{M}$ data blocks are superimposed to get the final signal $\mathrm{S}(\mathrm{t})$.

$$
S(t)=\sum_{k=0}^{M-1} S^{m}(t) ; 0 \leq t \leq M T+L-\frac{T}{2}
$$

Combining formulas 2 and 4 , we can get the formula 5

$$
S(t)=\sum_{m=1}^{M} \sum_{k=0}^{N-1}\left[a_{k}^{m} h(t-m T)+j b_{k}^{m} h(t-T / 2-m T)\right] e^{j\left(\frac{2 \pi}{T}+\frac{\pi}{2}\right) k}, k=0,1, \ldots ., N-1
$$

Fig. 3 shows the OQAM post-processing in FBMC for k even \& odd. The Synthesis Filter Bank (SFB) is used in the modulator region. The Offset QAM is one of the information sources for the SFB. The IFFT piece essentially depicts the subcarrier frequency shift. From the standpoint of the equipment, it conducts the calculations in a piece-by-piece manner. That is, a set of tests is pushed into the $\mathrm{N}$ branches of the IFFT obstruct on the double, and a set of yield tests is generated. After the polyphase filters, an element of $\mathrm{N} / 2$ is used to perform upsampling. The following examples from the parallel branches undergo a parallel-to-serial transition thanks to a unique combination of deferrals and adders. The demodulator, also known as the Analysis Filter Bank (AFB), is a component of the demodulator. The OQAM symbols are the outputs of the parallel branches, and they must go through OQAM post-processing, which reverses the procedure [5].

\section{The Prototype PHYDYAS Filter Design}

PHYDYAS stands for Physical Layer for Dynamic Access and Cognitive Radio. It offers the FBMC as the physical layer for dynamic access spectrum management (DASM) and cognitive radio communications. The proposed PHYDYAS filter is defined as follows [10]:

$$
h_{p}(t)=1+2 \sum_{k=1}^{k-1}\left(b_{k} \cos (2 \pi k / K T)\right.
$$


where $1 / \mathrm{T}$ is the subcarrier frequency separation and the coefficients $b_{k}$ satisfy the relation $b_{K}^{2}+b_{K-k}^{2}=1$ so that the filter satisfies Nyquist condition. For $\mathrm{K}=4$ the coefficients are $\mathrm{b} 1=0.97196, b 2=1 / 2$, and $b 3=\sqrt{1-b_{1}^{2}}$. The reader can go to for additional $\mathrm{K}$ values. It can be shown that $h_{p}(t)$ has a periodic period of KT, which is sampled with $\mathrm{L}=\mathrm{KM}$ samples. The PHYDYAS filter has a unique property in that the shaping can be done in the frequency domain, that is, before the IFFT operation, by convolving the set of coefficients bk with the modulating symbols and then applying. However, this would need the use of KM points. In the case of the polyphase network (PPN) implementation for the shaping filter, instead of $\mathrm{M}$ point IFFT, as is the case for the other types of shaping filters, IFFT is used instead of M point IFFT [10].

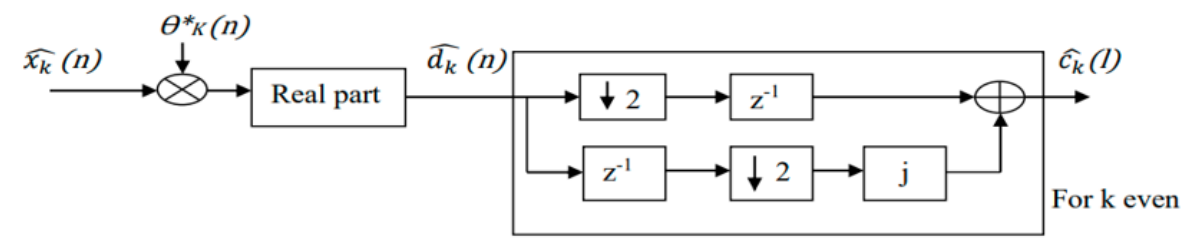

Real to complex conversion

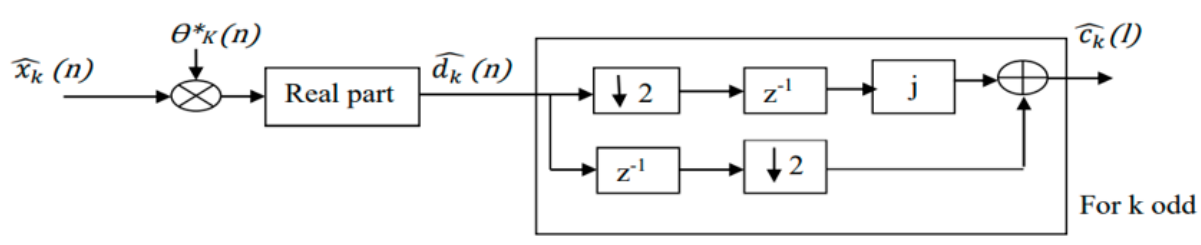

Real to complex conversion

Figure 3: OQAM post-processing in FBMC for $\mathrm{k}$ even and odd

The Nyquist principle dictates that the transmission filter's impulse response must cross the zero axes at all integer multiples of the symbol duration. The symmetry condition about the cut-off frequency, which is half the symbol rate, converts the condition into the frequency domain. After that, considering the frequency coefficients and imposing the symmetry condition is a simple way to construct a Nyquist filter. The global Nyquist filter is typically split into two sections in transmission systems: a half-Nyquist filter in the transmitter and a half-Nyquist filter in the receiver. The squares of the frequency coefficients then satisfy the symmetry condition. The frequency coefficients of the half-Nyquist filter obtained for $\mathrm{K}=2,3$ and 4 are given in Table $\mathrm{I}$.

TABLE I

Frequency Domain Prototype Filter Coefficients [11]

\begin{tabular}{|c|c|c|c|c|}
\hline $\mathrm{K}$ & $\mathrm{H} 0$ & $\mathrm{H} 1$ & $\mathrm{H} 2$ & $\mathrm{H} 3$ \\
\hline 2 & 1 & $\sqrt{2} / 2$ & - & - \\
\hline 3 & 1 & 0.911438 & 0.411438 & - \\
\hline 4 & 1 & 0.971960 & $\sqrt{2} / 2$ & 0.235147 \\
\hline
\end{tabular}

The filter response in the frequency domain consists of $2 \mathrm{~K}-1$ pulses, as shown in Fig. 4 for $\mathrm{K}=4$. The interpolation formula for sampled signals yields the continuous frequency response as shown in Fig. 5. Out-of-band ripples have almost vanished, and a highly selective filter has been achieved. 


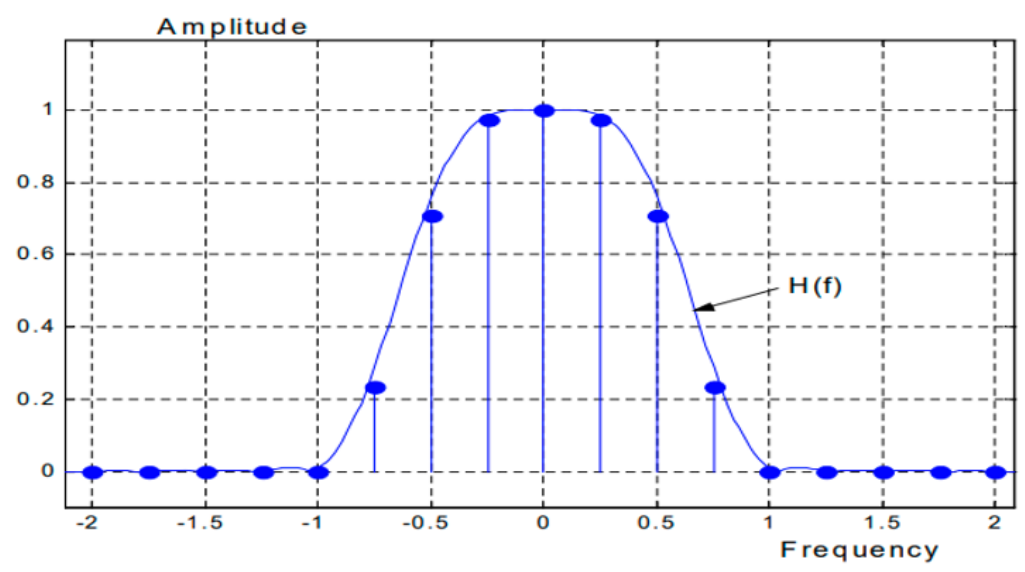

Figure 4: Prototype filter frequency coefficients and frequency response for $\mathrm{K}=4$ [11]

Fig. 5 shows the impulse response of the filter when length $\mathrm{L}=1024$, the number of sub-channels $\mathrm{M}=256$ and $\mathrm{K}=4$. The resultant set of $\mathrm{M}$ samples is referred to as a multicarrier tag, with $\mathrm{m}$ denoting the symbol index. A parallel-to-serial (P/S) converter is added to the IFFT's output for channel transmission, and the samples $x(n)$ appear in serial form. The transmitted signal has a sampling frequency of unity, $\mathrm{M}$ carriers, and a carrier frequency spacing of $1 / \mathrm{M}$. A multicarrier symbol's length $\mathrm{T}$ equals $\mathrm{M}$, which is the opposite of carrier spacing. $\mathrm{T}$ is also the multicarrier symbol duration, which represents the fact that in the time domain, successive multicarrier symbols do [11].

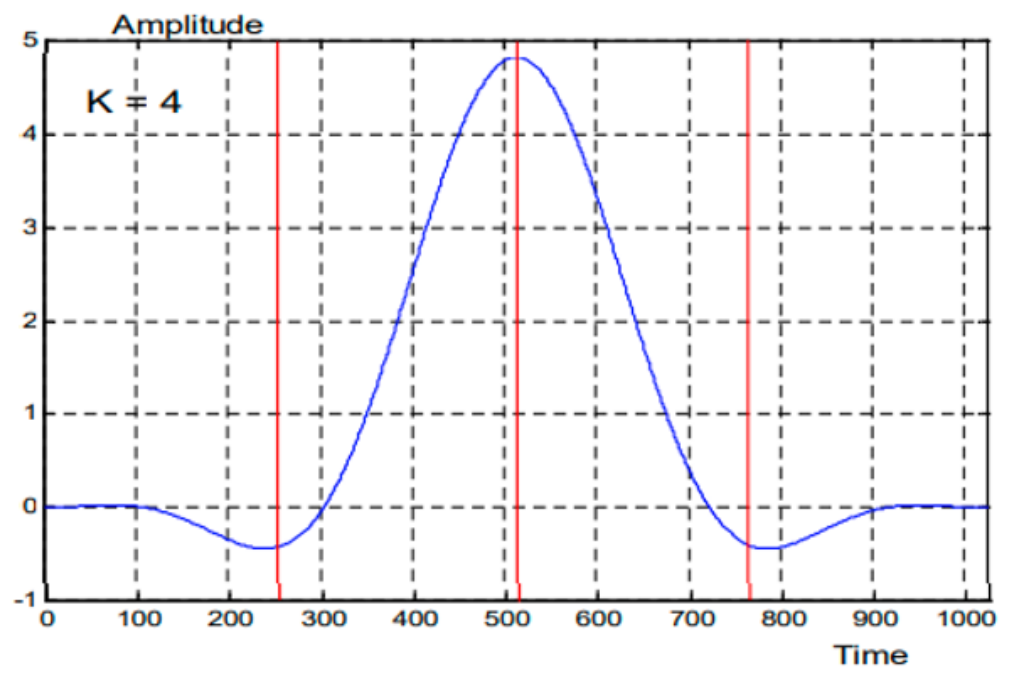

Figure 5: Impulse response of the prototype filter for overlapping factor $\mathrm{K}=4$ [11]

\section{Simulation of FBMC System}

To evaluate the performance of the FBMC system, a simulation model using $\mathrm{m}$-files via MATLAB design package is designed with the block diagram shown in Fig. 6. The data are generated as 600 frames each frame of size 64 bits. The 
QAM modulation used is 16-QAM. In the OQAM modulator, real and imaginary parts are alternated. In upsampling, the data are upsampled by K (overlapping factor=4) then using PHYDYAS shaping filter. On the receiver side, the operation in the FBMC receiver is the opposite of the operation in the FBMC transmitter as shown in Fig. 6(b). The detection type used is the maximal likelihood. No channel coding or equalization is used and perfect synchronization between transmitter and receiver is assumed. One channel is used in the simulations, this is a Stanford University Interim (SUI) Channel which is a set of six-channel models that represent three terrain types. The model used in the tests is SUI-6 whose characteristics are shown in Table II.

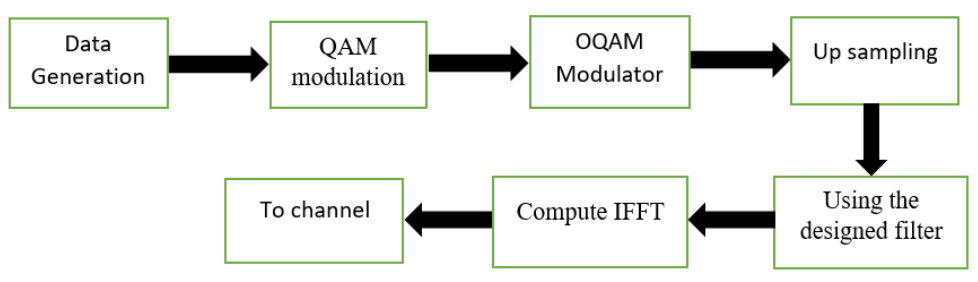

(a)

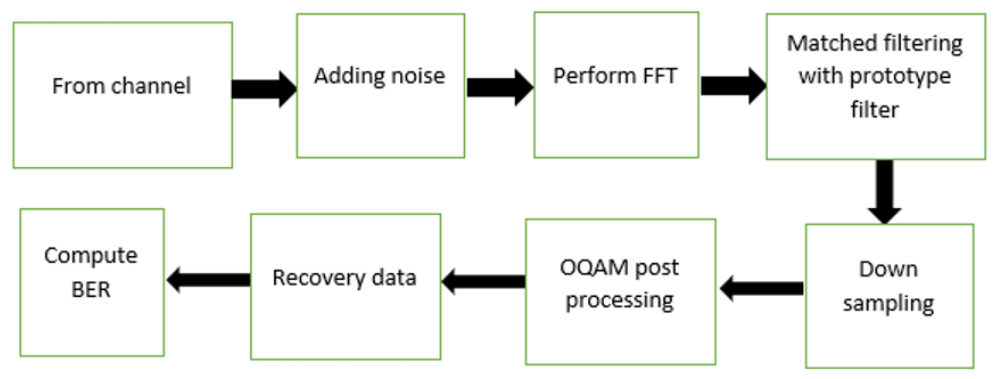

(b)

Figure 6: Block diagram of simulated FBMC System (a)Transmitter, (b) Receiver

TABLE II

SUI-6 Channel Model [12]

\begin{tabular}{|c|c|c|c|c|}
\hline Parameter & Tap 1 & Tap 2 & Tap 3 & Units \\
\hline Delay & 0 & 14 & 20 & $\mu \mathrm{s}$ \\
\hline Power (omni ant.) & 0 & -10 & -14 & $\mathrm{~dB}$ \\
\hline 90\% K-factor (omni) & 0 & 0 & 0 & \\
\hline $75 \%$ K-factor (omni) & 0 & 0 & 0 & \\
\hline $50 \%$ K-factor (omni) & 1 & 0 & 0 & \\
\hline Power (30 ${ }^{\circ}$ ant.) & 0 & -16 & -26 & $\mathrm{~dB}$ \\
\hline $90 \%$ K-factor $\left(30^{\circ}\right)$ & 0 & 0 & 0 & \\
\hline $75 \%$ K-factor $\left(30^{\circ}\right)$ & 2 & 0 & 0 & \\
\hline $50 \%$ K-factor $\left(30^{\circ}\right)$ & 5 & 0 & 0 & \\
\hline Doppler & 0.4 & 0.3 & 0.5 & $\mathrm{~Hz}$ \\
\hline
\end{tabular}

\section{Simulation Results}

To evaluate the performance of the designed FBMC system compared to OFDM system, two simulation models are implemented: FBMC and OFDM via MATLAB simulations under AWGN and SUI-6 multipath fading channels with two 
sets of simulation scenarios. Tables III and IV show the first set of simulation parameters of the designed OFDM and FBMC respectively. As shown in these tables although not all simulation parameters have the same values due to different system structures, they have the same action to provide fair comparisons.

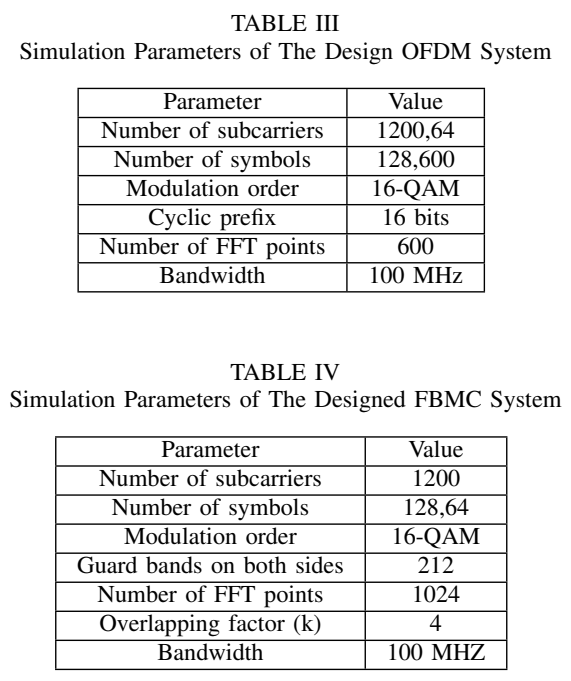

Fig. 7 shows the Bit error rate versus SNR for OFDM and FBMC systems in the AWGN channel. It can be seen in this figure that low SNR values (up to about $\mathrm{SNR}=8 \mathrm{~dB}$ ), OFDM and FBMC are very close in Bit error rate performance. At higher SNR values, OFDM has a relatively better Bit error rate compared to FBMC. Fig. 8 shows BER versus SNR for both OFDM and FBMC systems in the SUI-6 multipath fading channel. It can be seen in this figure the BER is almost comparable

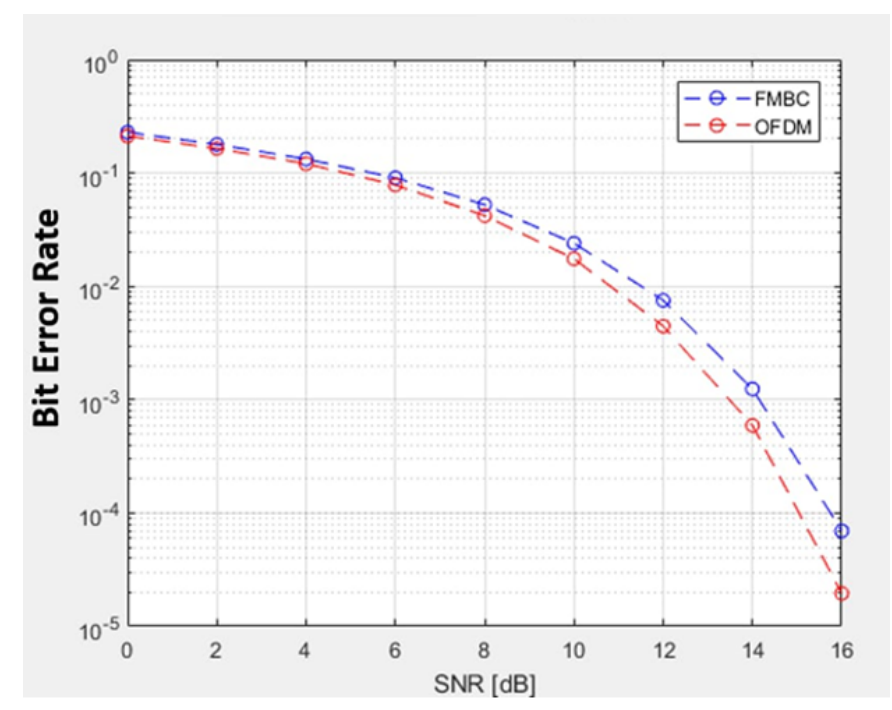

Figure 7: Bit error rate of OFDM and FBMC in AWGN channel 


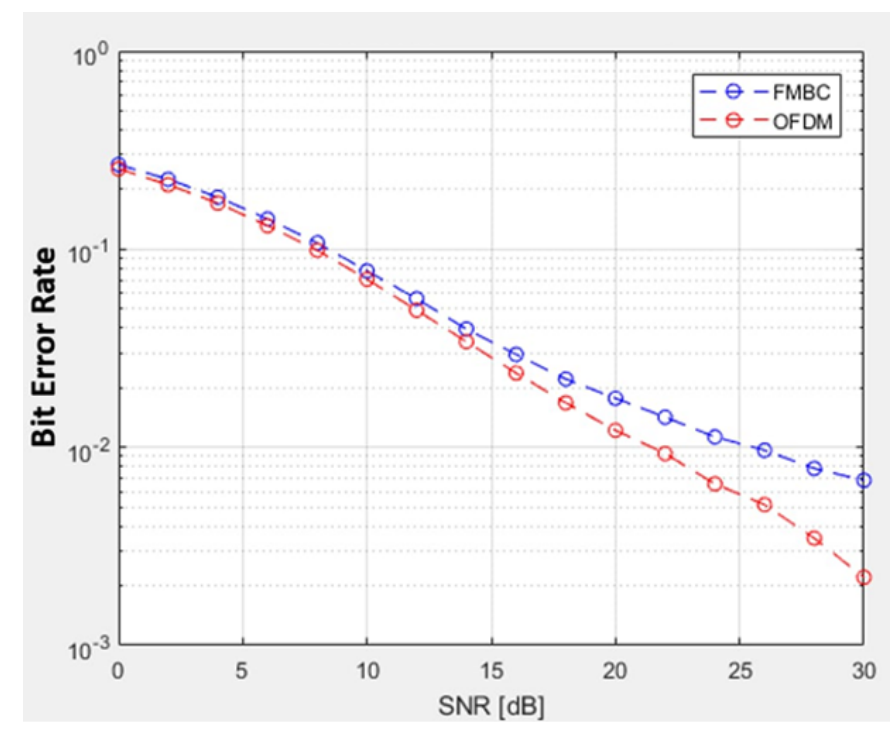

Figure 8: Bit error rate of OFDM and FBMC in SUI-6 channel

for both systems at low SNR values less than $10 \mathrm{~dB}$ at higher SNR values, it is clear that as SNR is increased the performance of FBMC is deteriorated compared to OFDM. Fig. 9 and Fig. 10 show BER versus SNR for OFDM and FBMC systems in AWGN and SUI-6 channels respectively when the number of subcarriers is reduced to 64 and the number of symbols is increased to 600 in OFDM but the number of symbols is reduced to 64 in FBMC. It can be seen in both figures that recognizable improvement in BER is introduced by FBMC compared to OFDM at SNR values less than 14 $\mathrm{dB}$ at higher SNR values, the improvement margin is significantly reduced.

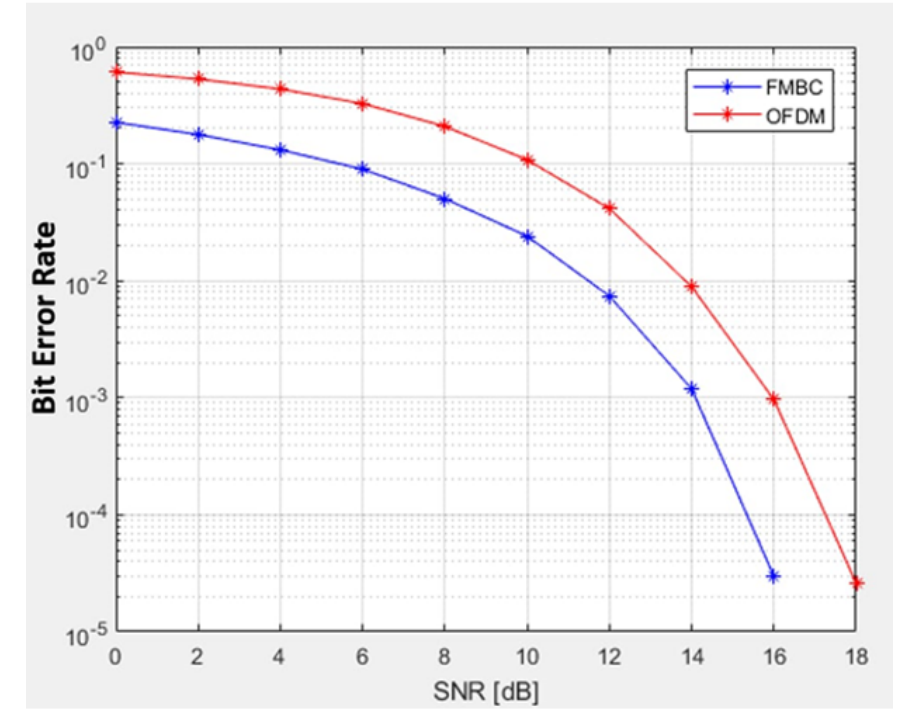

Figure 9: Bit error rate of OFDM and FBMC in AWGN channel 


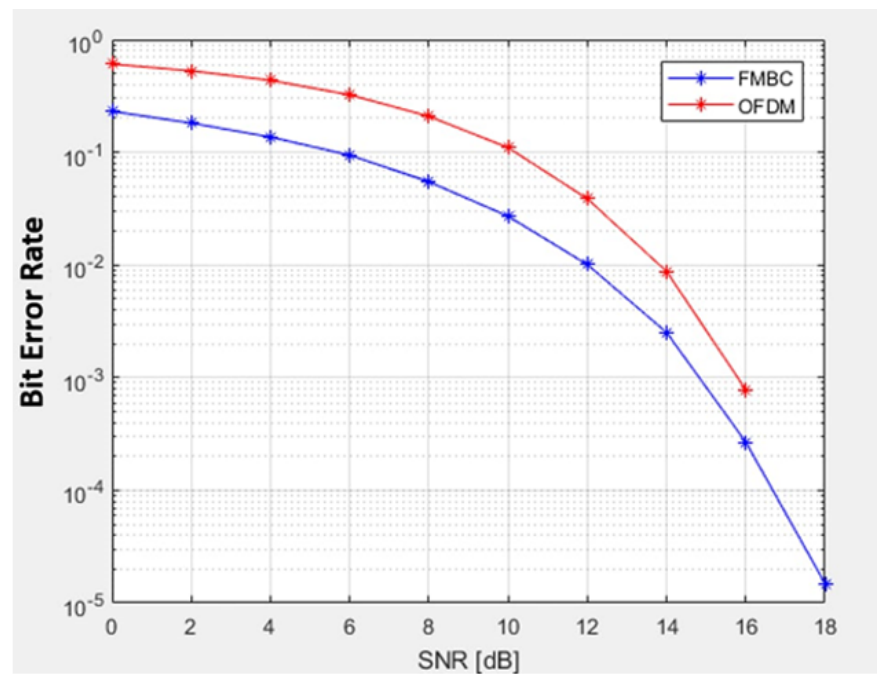

Figure 10: Bit error rate of OFDM and FBMC in SUI-6 channel

\section{Conclusion}

The paper presented performance comparisons between FBMC and OFDM under AWGN and SUI-6 multipath fading channels. In terms of spectral performance, FBMC is a better alternative to OFDM because it does not require CP and instead relies on a carefully designed pulse-shaping filter to reduce the effect of the multipath channel. As shown by the simulation results, OFDM and FBMC provide comparable performance in AWGN and SUI-6 channels when a high number of subcarriers is used. FMBC over-performs OFDM when the channel conditions become more severe. The amount of improvement is increased when the number of subcarriers used in both systems is decreased. The possible future works can be making comparisons when filter types other than PHYDYAS are used in terms of bit error rate and peak to average power ratio.

\section{REFERENCES}

[1] Kollar, Z, and Al-Amaireh, H, "FBMC Transmitters with Reduced Complexity" , Radio Engineering, Vol. 27, No. 4, 2018.

[2] Baker, K, and Quinley, J, "National Tribal Broadband Summit" , pp. 23-24, Washington, DC, September, 2019.

[3] Almeida, I. B, "GFDM as a Framework for FBMC-OQAM: Iterative Detection and Performance Evaluation" , Master thesis, Dresden Technical University, Germany, 2018.

[4] Kassim, M, "Filter Bank Multicarrier Modulation for 5G Mobile Communication and Cognitive Radio Based on SDR" , Doctoral dissertation, University of Technology, Iraq, 2020.

[5] Kaur, S, "Analysis of FBMC as a Candidate Waveform for 5G Networks", International Journal of Pure and Applied Mathematics, Vol. 118, No. 7, 2017.

[6] Yao, L, Wang, E, \& Peng, X, "Design and Research on FBMC-OQAM Multicarrier Technology for 5G" , In Journal of Physics: Conference Series, Vol. 1213, No. 5, p. 052068, pp. 1-7, IOP Publishing, 2019.

[7] Payaro, M, Pascual-Iserte, A, \& Najar, M, "Performance Comparison Between FBMC and OFDM in MIMO Systems Under Channel Uncertainty" , In European Wireless Conference (EW), pp. 1023-1030, Lucca, Italy, 2010.

[8] Zafar, A, "Filter Bank Based Multicarrier Systems for Future Wireless Networks" , Doctoral dissertation, University of Surrey, UK, 2018.

[9] Hu, J, Yang, J, Chuan, Y, \& Li, E, "Performance Analysis of OFDM and FBMC" , In IOP Conference Series: Materials Science and Engineering: Conference Series, Vol. 677, No. 3, p. 032001, pp. 1-6, IOP Publishing, 2019.

[10] Al-Haddad, M. K, \& Ziboon, H. T, "Comparative Study of Pulse Shaping Filters in FBMC" , Iraqi Journal of Computers, Communication, Control and Systems Engineering, Vol. 19, No. 2, pp. 30-40, 2019.

[11] Bellanger, M, Le Ruyet, D, Roviras, D, Terre, M, Nossek, J, Baltar, L, \& Ihaalainen, T,2010, "FBMC physical layer: A Primer" , Phydyas, Vol. 25, No. 4, pp. 7-10.

[12] Jain, R, "Channel Models: A Tutorial" , In WIMAX forum, AATG, Vol. 10, 2007. 